\title{
Stability and control of planar compass gait walking with series-elastic ankle actuation
}

\author{
Deniz Kerimoğlu', Ömer Morgül' and Uluç Saranl'
}

\begin{abstract}
Passive dynamic walking models are capable of capturing basic properties of walking behaviours and can generate stable human-like walking without any actuation on inclined surfaces. The passive compass gait model is among the simplest of such models, consisting of a planar point mass and two stick legs. A number of different actuation methods have been proposed both for this model and its more complex extensions to eliminate the need for a sloped ground, balancing collision losses using gravitational potential energy. In this study, we introduce and investigate an extended model with serieselastic actuation at the ankle towards a similar goal, realizing stable walking on level ground. Our model seeks to capture the basic structure of how humans utilize toe push-off prior to leg liftoff, and is intended to eventually be used for controlling the ankle joint in a lower-body robotic orthosis. We derive hybrid equations of motion for this model, and show numerically through Poincaré analysis that it can achieve asymptotically stable walking on level ground for certain choices of system parameters. We then study the bifurcation regimes of period doubling with this model, leading up to chaotic walking patterns. Finally, we show that feedback control on the initial extension of the series ankle spring can be used to improve and extend system stability.
\end{abstract}

\section{Keywords}

Dynamic walking, passive compass gait, series-elastic actuation, ankle actuation, bifurcation analysis, feedback control

\section{Introduction}

Legged locomotion is achieved through the coordination of numerous complex components within neuromuscular and skeletal systems. Modelling such behaviours by considering these systems in their entirety would result in excessively complex and impractical mathematical models, making it impossible to introduce general methods for their analysis and control. On the other hand, simpler models that abstract away unnecessary details and focus on basic characteristics of particular behaviours can provide better foundations for analysis and control.

In the context of bipedal locomotion, passive dynamic walking models provide successful examples of this approach. In particular, the passive compass gait (PCG) is the simplest of such models (Collins et al., 2001; Garcia et al., 1996, 1998; McGeer, 1990), capturing the basic structure and dynamics of human walking with those of a simple double pendulum motion, combined with an instantaneous switch of leg support. This model can achieve asymptotically stable, humanlike walking on slightly sloped ground, powered only by gravity and lacking any explicit controls. The PCG model is uniquely useful since it is structurally simple and capable of generating human-like walking without any actuation. Our motivation for studying the PCG model and extending it with series elastic actuation is hence to exploit its passive dynamics to simplify stable control of walking, while also achieving energy efficiency. In general, purely passive PCG models can only provide very narrow basins of attraction, and require a sloped ground to recover the energy lost to collisions with the ground (Goswami et al., 1996). Both of these problems have been addressed to some extent by various researchers in the literature, eliminating the need for a sloped ground and extending stability by introducing active feedback control. For example, Goswami et al. (1996, 1997) used passivity mimicking control with hip and ankle torque actuation to obtain slope invariant walking even on positive slopes. Kuo (2002) used impulsive input along the support leg immediately before heel strike together with torque on the support leg to achieve energetically efficient walking. To achieve slope invariance and to increase the basin of attraction, Spong and Bhatia (2003) considered passivity-based control, using total energy shaping, increasing the robustness of the system to external disturbances and variations in ground slope. Dynamic locomotion on rough terrain with a minimalistic control approach was investigated in Byl and Tedrake (2008)

\footnotetext{
'Department of Electrical and Electronics Engineering, TR-06800 Bilkent, Ankara, Turkey

${ }^{2}$ Department of Computer Engineering, Middle East Technical University, Universiteler Mah. Dumlupinar Blv. No: I, 06800, Ankara, Turkey

\section{Corresponding author:}

Deniz Kerimoğlu, Department of Electrical and Electronics Engineering, TR-06800 Bilkent, Ankara, Turkey.

Email: kerimogl@ee.bilkent.edu.tr
} 
and Iida and Tedrake (2009), where the authors also reported experimental results showing that their platform could achieve walking on uneven terrain.

Several studies on the compass gait also proposed methods of actuation through an ankle mechanism. For example, Ahn and Hogan (2012) and Maykranz and Seyfarth (2014) considered including an explicit model of the foot acting as a lever arm to provide thrust to the body through the leg. Extending the capabilities of the PCG model on uneven terrain using a torque at the hip and an impulsive toe-off control was investigated in Byl and Tedrake (2008). Impulsive input applied at toe-off immediately before heel strike was also studied separately by Bhounsule (2015) and Kuo (2002). Van Der Linde (1998) extended the PCG model with radial spring actuation on the stance leg, activated during mid-stance by instantaneously changing its stiffness. Asano et al. (2005) considered parametric excitation using telescopic leg actuation with spring and damper to obtain walking on level ground. Zelik et al. (2014) developed a simple dynamic walking model with feet and series elasticity at the ankle joint. The authors show that the trailing leg push-off which starts before the collision of the leading leg, reduces collision losses. Ghorbani (2008) introduced a spring mechanism on the ankle joint of a PCG with foot in order to store part of the energy during the collision and to release it during the double stance phase passively. A three degree of freedom (DOF) spatial PCG model with under-actuated ankles and with only one actuator in the hip joint is considered in Tang et al. (2014). The authors have obtained an open loop limit cycle and stabilized this limit cycle utilizing a discrete-time linear quadratic regulator (DLQR) method which was shown to have a larger basin of attraction. Wang et al. (2015) introduced a compass model with foot by including a constraint mechanism in the hip joint and rotary springs in the ankles which can walk with a minimal cost of transport. Tedrake et al. (2004) presented a three dimensional dynamic walker platform, which is actuated by adding two active joints on the ankle to generate pitch and roll motions. Hobbelen and Wisse (2008a) studied the effects of ankle actuation on energy expenditure, disturbance rejection and the versatility of passive walking with the use of two mathematical models and one physical walker prototype. Collins and Ruina (2005) presented an autonomous, three dimensional bipedal walking robot with efficient and humanlike motions, consisting only of ankle actuation through a spring. Hobbelen et al. (2008b) developed energy-effective humanoid robots which utilize series elasticity at knee and ankle joints. Goswami et al. (1998) studied symmetric and steady stable gaits of the PCG model, which gradually evolves through a regime of bifurcations and eventually exhibits an apparently chaotic gait. Finally, Garcia et al. (2000) analyzed walker designs with and without knees, as well as with different foot structures to study bifurcation regimes of a passive kneed walker.

In this paper, we focus on the effects of adding serieselastic actuation (SEA) on the ankle on PCG model gaits and their stability. Motivated by the compliance, efficiency and high bandwidth properties of animal muscle and tendons, SEA platforms are used in developing actuated assistive orthoses and prostheses. For example, Hitt et al. (2007) developed a portable actuated ankle-foot orthosis (AFO) that can actively support locomotion for partially limited ambulators during daily activities. Blaya et al. (2004) used a force controllable SEA to control the impedance of the orthotic ankle joint throughout the walking cycle to treat the drop-foot gait disorder. Hitt et al. (2009) developed an active ankle foot prosthesis (AAFP) which utilizes SEA to prevent the need for a large and heavy impedance-controlled motor. Using both series and parallel elasticity, Au et al. (2009) developed an AAFP that fulfils the demanding human-like ankle specifications and decreases metabolic cost of amputee locomotion compared to a conventional passive-elastic prostheses.

Previously, in Kerimoglu et al. (2015) we had introduced the ankle actuated model and studied dependence of behaviour on only the spring stiffness and investigated the stability and existence of fixed points, but had not performed bifurcation analysis and feedback control. However, in this study we extend our previous work by presenting bifurcation analysis and feedback control based on ankle spring precompression.

In general, studies in the literature on ankle actuation for the planar PCG model utilizing non-instantaneous spring thrust phases are mainly concerned with the efficiency of walking. Van Der Linde (1998) and Asano et al. (2005) considered adding a compliant telescopic leg with variable spring to the PCG model to compensate for collision losses by triggering spring in single stance phase and investigated open loop stable gaits. In our present study, however, we model human ankle behaviour by actuating the spring through a double stance phase. Efficient walking platforms with series elasticity at the ankle were developed by Collins and Ruina (2005), which considered an open loop control of the platform and Hobbelen and Wisse (2008a), where a seven DOF platform was actuated and the effect of rotary spring stiffness on energy expenditure through a simple PCG model with feet was studied. In our study, we develop a simpler model with point foot and focus on gaits that are controlled as both open and closed-loop systems as a function of spring precompression, chosen intentionally to better capture series elasticity. Several additional studies are concerned with providing explanations for the human ankle behaviour. For example, Maykranz and Seyfarth (2014) extended the spring-loaded inverted pendulum (SLIP) model with a foot and a compliant ankle to investigate human running behaviour, which was reported to exhibit asymmetries between touch-down and take-off. Zelik et al. (2014) introduced a walking model with a series elastic ankle, modelling the Achilles tendon and investigating the effects of ankle elasticity on the walking economy by adjusting the ratio between ankle stiffness and foot length as well as push-off timing through ankle and hip actuation. Ahn and Hogan (2012) analyzed the effects of torsional ankle impedance on a PCG model with feet and reported that impedance on the ankle yields more stable walking than directly using ankle torque with zero impedance. We differ from these last three studies in terms of modelling, through our use of series elastic actuation, analysis, with extensive results on stability and period doubling bifurcations, and finally control, where we propose feedback control on spring precompression towards stable and controllable walking. There are a number of related studies in the literature that also apply feedback control to the PCG model with compliant ankle joints. For example, Ghorbani (2008) introduced a simplified model of 
bipedal walking with adjustable stiffness artificial tendon in the colliding ankle joint with a linear spring on the trailing leg, emulating the force generated by the trailing leg during the double support phase. The authors reduced energy lost to collisions by adjusting the stiffness of the colliding ankle and controlled energy injected into the biped by adjusting the stiffness of the trailing leg. The modelling approach developed in this study was intended to actuate bipedal walking robots and was mainly interested in adjustable stiffness. In our study, however, we model SEA for the purpose of developing Active Ankle Foot Orthosis (AAFOs) where we considered the precompression as the parameter of interest. Wang et al. (2015) extended the PCG model by introducing a constraint mechanism in the hip joint and rotary springs in the ankles, achieving highly efficient but unstable walking gaits. Through transverse coordinate control, the authors obtained both stable and efficient walking. Our study differs from this study in terms of the underlying model, the extent of our parameter dependent stability analysis and our active closedloop feedback control on spring precompression.

In relation to the literature summarized above, novel contributions of our paper are threefold. First, we model the action of a SEA mechanism on the PCG model by adding a radial spring at the ankle, obtaining stable walking on level ground for large ranges of model parameters. Seeking to model the function of human ankle, the proposed model is simpler than alternatives in the literature, with only a linear spring and point foot to provide thrust during a noninstantaneous toe push-off phase. The spring is assumed to be compressed slowly during the stance phase and released immediately after heel strike. Second, we thoroughly explore and investigate the effect of ankle spring parameters on walking by deriving the hybrid dynamics of the model, finding and characterizing the stability of fixed points and performing parameter dependent stability analysis via Poincaré methods. In a real platform, since SEA would be used to adjust the precompression of the spring, we focus our analysis on the dependence of stability on this spring precompression parameter across a very large range. As we increase the amount of precompression, we inject more energy into the model and observe stable, period $2^{n}$ bifurcations, chaotic and eventually unstable gaits. This study on energy injection into the model assumes the precompression to be chosen in an open-loop manner, and hence does not yield stable gaits for all velocities. Finally, to address this issue, we propose an active feedback controller on the spring precompression which can stabilize gaits that were previously period $2^{n}$, chaotic and unstable gaits, reducing them to regular, period-one walking gaits. The proposed controller uses the forward velocity feedback to perform once-per-step adjustments on the spring precompression. Computing the eigenvalues of the return map Jacobian, we show that the controller not only improves the stability of the walking model across a large range of precompression values, but also provides a control policy to achieve, robust walking for desired speed and step length values from a large range.

This paper is organized as follows. In the next section, we introduce our serially-actuated compass gait model, describing its underlying assumptions and the resulting equations of motion. In the section 'Stability of walking with the AACG model', we use Poincaré methods to first identify limit cycles of the model for a specific choice of model parameters, and subsequently perform parameter dependent stability analysis leading to our investigation of bifurcation regimes of the model. Finally, the section 'Feedback control through the ankle spring' introduces our proposed feedback control on the spring precompression to stabilize otherwise unstable limit cycles, and we draw our conclusions in the final section.

\section{The ankle-actuated compass gait model}

\section{Modelling assumptions}

During normal human walking, the ankle torque is transmitted to the leg through the lever arm of the foot and the resulting force on the body depends on the internal dynamics of the leg. Nevertheless, a sufficiently accurate model can still be obtained if the masses of both the leg and the toe, together with ankle kinematics are assumed to be negligible, allowing a direct model of the ankle torque as a radial force on the leg as shown in Figure 1. Based on this observation, our model will represent the foot as a point contact, and the action of ankle joint as a linear force component acting along the leg. The resulting serially-actuated active prismatic joint will then be used to compensate for energy losses due to ground collisions during toe push-off.

This assumption leads to our simplified model, which we call the ankle-actuated compass gait (AACG) model, shown in Figure 2. It consists of a point mass $M$ modelling the torso, to which two legs of length $l$ with small mid-length masses $m$ are attached. For the stance leg, we assume that a linear ankle spring with stiffness $k$ and rest length $r_{0}$ is available and can be released before the leg lifts off. The length of the spring, $r(t)$ is constrained with $r(t)>0$, capturing the unidirectional nature of heel contact with the ground. The remaining configuration variables $\theta_{s}$ and $\theta_{n}$ represent the angles of the supporting and non-supporting leg angles relative to the vertical, respectively and $p_{\text {toe }}$ denotes the position of the non-stance toe. The scope of the present paper is limited to planar walking on flat ground, although our results can be generalized to constant but non-zero slopes as well.

For normal, steady-state walking, the AACG model is assumed to go through two phases, single support and double support, separated by an infinitesimal 'collision event' to

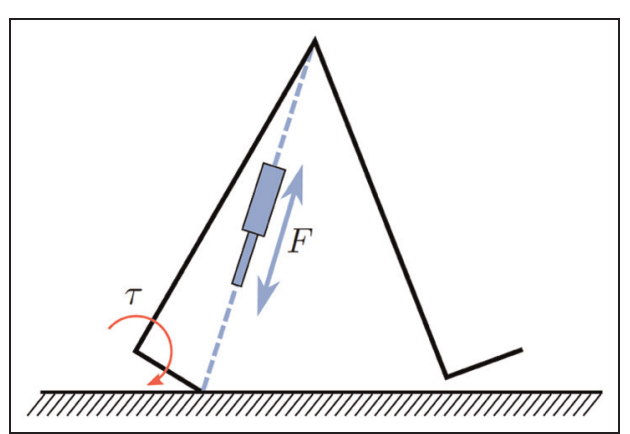

Figure I. Modelling of the action of ankle torque $\tau$ as a radial force $F$ on the body. 


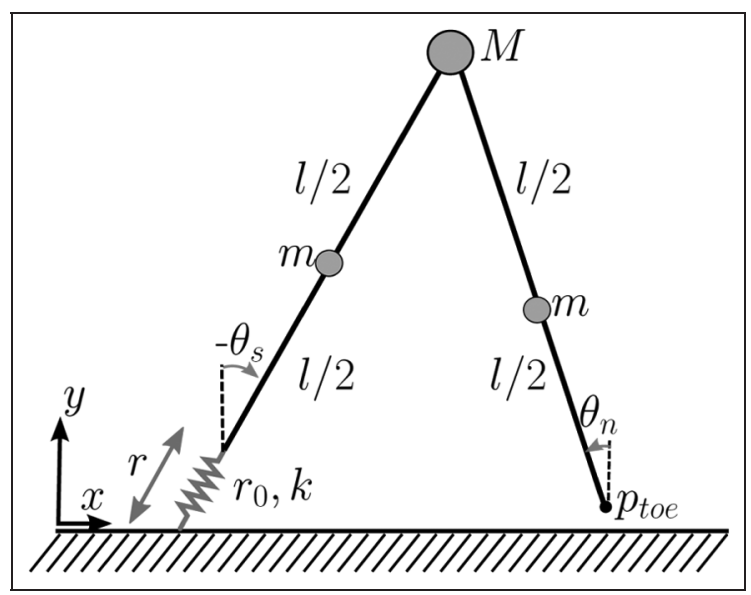

Figure 2. The ankle-actuated compass gait model.

capture the effects of weight transfer from one foot to the other as shown in Figure 3. During the single support phase, the AACG model exhibits the dynamics of a double pendulum similar to the standard PCG model. The supporting leg is in contact with the ground and the other leg is free to swing, with the stance leg spring assumed to be locked with $r=0$, capturing the effect of human heel contact with the ground. Similar to the standard PCG model, we ignore foot scuffing collisions occurring at the instance when swing leg passes stance leg. The single support phase ends when the swing leg comes into contact with the ground ahead of the stance leg.

Since the collision with the ground is slip-free and inelastic, it preserves configurations, but results in a discrete change in velocities due to impulsive collision forces. Following the collision, the standard PCG model performs an immediate weight transfer and resumes with the subsequent single support phase. The AACG model, however, follows the collision with a non-instantaneous double support phase, wherein both legs remain on the ground and a 'precompressed' ankle spring in series with the trailing stance leg is released. This results in a forward thrust supported by the fixed trailing toe, with the front leg pivoting freely around its newly acquired toe contact. The ankle spring continues to extend until it reaches its rest length (which we also refer to as the spring precompression), at which point the trailing stance leg lifts off and the spring is brought back to $r=0$ in preparation for the next toe push-off. The system then continues on to the next single support phase. Figure 3 depicts the two phases of the model as well as the collision event. The following sections present models associated with each phase.

\section{Dynamics of the single support phase}

The single support phase for the AACG model has the same structure as the PCG model, with identical equations of motion. We parameterize the two dimensional configuration space in this phase with the angles of the support and nonsupport legs to yield the definition

$$
\boldsymbol{q}_{s s}:=\left[\theta_{s}, \theta_{n}\right]^{\mathrm{T}}
$$

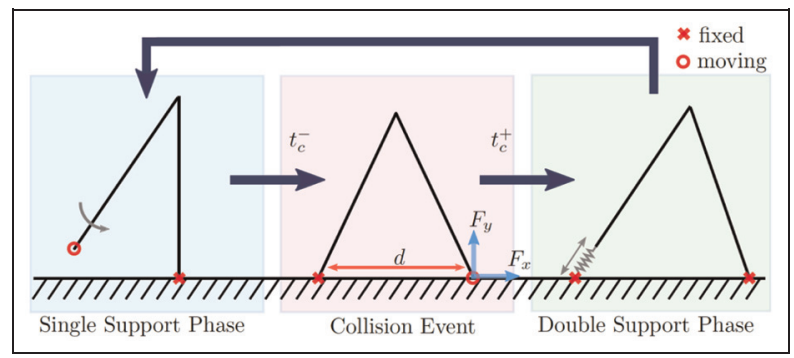

Figure 3. Hybrid phases of walking with the ankle-actuated compass gait model. The collision event is instantaneous and is modelled with a discontinuous change in system velocities.

Detailed derivations for the dynamics during the single support phase have been extensively covered in the literature, and will not be reproduced here. The reader can refer to Goswami et al. (1997) for details.

\section{The collision map}

When the swing leg collides with the ground, the AACG model experiences impulsive forces, resulting in an instantaneous change in system velocities, while the model configuration remains unchanged. However, unlike the standard PCG model, both legs remain fixed on the ground for the AACG model and the angular momentum of the system is no longer conserved around either toe. Consequently, we fall back to Lagrangian methods with impulsive forces, similar to the methods used in Grizzle et al. (2001), to derive the postcollision velocities. This method uses the unconstrained, three DOF AACG model with a released spring shown in Figure 2 and identifies impulsive constraint forces on the swing toe that would bring its velocity to zero.

The three dimensional configuration space of the system during this instantaneous collision phase can be defined as

$$
\boldsymbol{q}_{c}:=\left[\theta_{s}, \theta_{n}, r\right]^{\mathrm{T}}
$$

Impulsive forces experienced by the swing leg during the collision event are illustrated in the middle diagram in Figure 3. Writing the system kinetic and potential energy expressions as a function of $\boldsymbol{q}_{c}$, we first obtain the Lagrangian, which is then used to derive the equations of motion

$$
\mathbf{M}_{c}\left(\mathbf{q}_{c}\right) \ddot{\mathbf{q}}_{c}+\mathbf{B}_{c}\left(\mathbf{q}_{c}, \dot{\mathbf{q}}_{c}\right) \dot{\mathbf{q}}_{c}+\mathbf{G}_{c}\left(\mathbf{q}_{c}\right)=\mathbf{J}_{c}^{\mathrm{T}}\left(\mathbf{q}_{c}\right) \mathbf{F}_{I} \delta\left(t-t_{c}\right)
$$

that remain valid only instantaneously with $t \in\left[t_{c}^{-}, t_{c}^{+}\right]$. Here, the Dirac delta function $\delta\left(t-t_{c}\right)$ is used to represent the impulsive forces acting on the swing toe at $t=t_{c}$. The left side of the equation captures the continuous dynamics of the three DOF model, detailed derivations of which are omitted since the calculations are lengthy and straightforward.

$\mathbf{M}_{c}\left(\mathbf{q}_{c}\right)$ is the $3 \times 3$ mass matrix, $\mathbf{B}_{c}\left(\mathbf{q}_{c}, \dot{\mathbf{q}}_{c}\right)$ is $3 \times 3$ matrix which represents Coriolis forces and $\mathbf{G}_{c}\left(\mathbf{q}_{c}\right)$ is $3 \times 1$ vector which captures gravitational forces. The Jacobian transpose $\mathbf{J}_{c}^{\mathrm{T}}\left(\mathbf{q}_{c}\right)$ is a $3 \times 2$ matrix which maps velocities from swing toe coordinate to generalized coordinates and $\mathbf{F}_{I}=\left[F_{x}, F_{y}\right]^{\mathrm{T}}$ is a 
$2 \times 1$ vector which represents external impulsive forces along $x$ and $y$ axes acting on the system during collision.

Solving for system accelerations, we have

$$
\ddot{\mathbf{q}}_{c}=\mathbf{M}_{c}\left(\mathbf{q}_{c}\right)^{-1}\left(\mathbf{J}_{c}^{\mathrm{T}}\left(\mathbf{q}_{c}\right) \mathbf{F}_{I} \delta\left(t-t_{c}\right)-\mathbf{B}_{c}\left(\mathbf{q}_{c}, \dot{\mathbf{q}}_{c}\right) \dot{\mathbf{q}}_{c}-\mathbf{G}_{c}\left(\mathbf{q}_{c}\right)\right)
$$

where $\mathbf{M}_{c}\left(\mathbf{q}_{c}\right)$ is assumed to be invertible.

Model velocities before and after the collision are related through the integral of these dynamics, yielding

$$
\int_{t_{c}^{-}}^{t_{c}^{+}} \ddot{\mathbf{q}}_{c} d t=\int_{t_{c}^{-}}^{t_{c}^{+}} \mathbf{M}_{c}\left(\mathbf{q}_{c}\right)^{-1}\left(\mathbf{J}_{c}^{\mathrm{T}}\left(\mathbf{q}_{c}\right) \mathbf{F}_{I} \delta\left(t-t_{c}\right)-\mathbf{B}_{c}\left(\mathbf{q}_{c}, \dot{\mathbf{q}}_{c}\right) \dot{\mathbf{q}}_{c}-\mathbf{G}_{c}\left(\mathbf{q}_{c}\right)\right) d t
$$

where $t_{c}^{-}$and $t_{c}^{+}$represent time instants just before and after collision, respectively. Since $\mathbf{q}_{c}$ is differentiable and $\dot{\mathbf{q}}_{c}$ is continuous, the non-impulsive terms $\mathbf{B}_{c}\left(\mathbf{q}_{c}, \dot{\mathbf{q}}_{c}\right) \dot{\mathbf{q}}_{c}$ and $\mathbf{G}_{c}\left(\mathbf{q}_{c}\right)$ vanish in the infinitesimal integration which takes place from time $t_{c}^{-}$to $t_{c}^{+}$. Since configurations remain continuous, the integral on the left hand side also reduces to the difference in configuration velocities. Hence, the result of the integration is equal to the post collision velocities and the effects of the noninstantaneous variables are cancelled yielding the following simplified collision map

$$
\dot{\mathbf{q}}_{c}\left(t_{c}^{+}\right)-\dot{\mathbf{q}}_{c}\left(t_{c}^{-}\right)=\mathbf{M}_{c}\left(\mathbf{q}_{c}\left(t_{c}\right)\right)^{-1} \mathbf{J}_{c}^{\mathrm{T}}\left(\mathbf{q}_{c}\left(t_{c}\right)\right) \mathbf{F}_{I}
$$

where $\dot{\mathbf{q}}_{c}\left(t_{c}^{-}\right)$and $\dot{\mathbf{q}}_{c}\left(t_{c}^{+}\right)$denote system velocities just before and after the collision, respectively. For simplicity, we define $\dot{\mathbf{q}}_{c}\left(t_{c}^{-}\right)$as $\dot{\mathbf{q}}_{c}^{-}$and $\dot{\mathbf{q}}_{c}\left(t_{c}^{+}\right)$as $\dot{\mathbf{q}}_{c}^{+}$from now on. In order to find this unknown collision force, we impose the constraint that the swing toe must come to rest following the plastic collision. Forward kinematics yields the swing toe velocities as a function of the generalized coordinates as

$$
\left[\begin{array}{c}
\dot{x}_{\text {toe }} \\
\dot{y}_{\text {toe }}
\end{array}\right]=\left[\begin{array}{ccc}
r \cos \theta_{s} & -l \cos \theta_{n} & \sin \theta_{s} \\
-r \sin \theta_{s} & l \sin \theta_{n} & \cos \theta_{s}
\end{array}\right]\left[\begin{array}{c}
\dot{\theta}_{s} \\
\dot{\theta}_{n} \\
\dot{r}
\end{array}\right]=\mathbf{J}_{c}\left(\mathbf{q}_{c}\right) \dot{\mathbf{q}}_{c}
$$

The plastic collision requires that post-collision toe velocities become zero, which can be expressed with the constraint

$$
\mathbf{J}_{c}\left(\mathbf{q}_{c}\right) \dot{\mathbf{q}}_{c}^{+}=\mathbf{0}
$$

Combining (6) and (8), we have

$$
\mathbf{J}_{c}\left(\mathbf{q}_{c}\right)\left(\dot{\mathbf{q}}_{c}^{-}+\mathbf{M}_{c}\left(\mathbf{q}_{c}\right)^{-1} \mathbf{J}_{c}^{\mathrm{T}}\left(\mathbf{q}_{c}\right) \mathbf{F}_{I}\right)=\mathbf{0}
$$

which can be solved for $\mathbf{F}_{I}$, which yields the final solution for post-collision configuration velocities as

$$
\begin{aligned}
\dot{\mathbf{q}}_{c}^{+}= & \left(\mathbf{I}-\mathbf{M}_{c}\left(\mathbf{q}_{c}\right)^{-1} \mathbf{J}_{c}^{\mathrm{T}}\left(\mathbf{q}_{c}\right)\left(\mathbf{J}_{c}\left(\mathbf{q}_{c}\right)\right.\right. \\
& \left.\left.\mathbf{M}_{c}\left(\mathbf{q}_{c}\right)^{-1} \mathbf{J}_{c}^{\mathrm{T}}\left(\mathbf{q}_{c}\right)\right)^{-1} \mathbf{J}_{c}\left(\mathbf{q}_{c}\right)\right) \dot{\mathbf{q}}_{c}^{-}=\mathbf{H}_{c}\left(\mathbf{q}_{c}^{-}\right) \dot{\mathbf{q}}_{c}^{-}
\end{aligned}
$$

Here, we assume that $\mathbf{J}_{c}\left(\mathbf{q}_{c}\right) \mathbf{M}_{c}\left(\mathbf{q}_{c}\right)^{-1} \mathbf{J}_{c}^{\mathrm{T}}\left(\mathbf{q}_{c}\right)$ is invertible. We note that $\mathbf{M}_{c}\left(\mathbf{q}_{c}\right)$ is a symmetric matrix which is assumed to be invertible. Hence, we have $\operatorname{rank}\left(\mathbf{J}_{c}\left(\mathbf{q}_{c}\right) \mathbf{M}_{c}\left(\mathbf{q}_{c}\right)^{-1}\right.$ $\left.\mathbf{J}_{c}^{\mathrm{T}}\left(\mathbf{q}_{c}\right)\right)=\operatorname{rank}\left(\mathbf{J}_{c}\left(\mathbf{q}_{c}\right)\right)$. From (7), it easily follows that when $\theta_{s}-\theta_{n} \neq \pi / 2$ or $r \neq 0$, we have $\operatorname{rank}\left(\mathbf{J}_{c}\left(\mathbf{q}_{c}\right)\right)=2$, which implies that $\mathbf{J}_{c}\left(\mathbf{q}_{c}\right) \mathbf{M}_{c}\left(\mathbf{q}_{c}\right)^{-1} \mathbf{J}_{c}^{\mathrm{T}}\left(\mathbf{q}_{c}\right)$ is invertible. Since these conditions always hold in our system, it follows that $\mathbf{J}_{c}\left(\mathbf{q}_{c}\right) \mathbf{M}_{c}\left(\mathbf{q}_{c}\right)^{-1} \mathbf{J}_{c}^{\mathrm{T}}\left(\mathbf{q}_{c}\right)$ is also always invertible.

The detailed expressions for the general form of $\mathbf{H}_{c}$, which is a $3 \times 3$ matrix capturing a general collision map with arbitrary initial states for the ankle spring, are given in the Appendix. In this paper, we focus on the AACG model with the ankle spring activated right before the collision, meaning that we have $r^{-}=0$ and $\dot{r}^{-}=0$. This simplifies the collision map to

$$
\dot{r}^{+}=\frac{2 m l \sin \left(\theta_{s}^{-}-\theta_{n}^{-}\right) \dot{\theta}_{n}^{-}-(3 m+4 M) \sin \left(2\left(\theta_{s}^{-}-\theta_{n}^{-}\right)\right) \dot{\theta}_{s}^{-}}{7 m+8 M+3 \cos \left(2\left(\theta_{s}^{-}-\theta_{n}^{-}\right)\right)}
$$

which is the form we use for all our simulations. Note that we only need to compute $\dot{r}^{+}$since the system has only a single DOF, the spring length, during double stance.

\section{Dynamics of the double support phase}

During the double support phase, both legs maintain contact with the ground and the ankle spring for the trailing leg spring is activated, resulting in a model with only the single, prismatic ankle DOF, $r(t)$. The remaining joint variables are constrained by the closed kinematic chain of the legs as shown in Figure 2. The single dimensional configuration space associated with the double support phase is hence defined as

$$
\mathbf{q}_{d s}:=r
$$

The leg angles, $\theta_{s}$ and $\theta_{n}$, are kinematically related to the ankle extension with

$$
\begin{aligned}
& \theta_{s}=-\pi / 2+\arccos \left(\frac{d^{2}-l^{2}+(l+r)^{2}}{2 d(l+r)}\right) \\
& \theta_{n}=\pi / 2-\arccos \left(\frac{d^{2}+l^{2}-(l+r)^{2}}{2 d l}\right)
\end{aligned}
$$

where $d$ is the distance between the toes, fixed at its value initialized with the preceding collision.

This phase continues until the ankle spring becomes fully extended, having transferred all of its potential energy into the system. At that point, the double support phase ends and the spring length is brought back to its precompressed state with $r=0$ in preparation for the next collision. The prismatic joint is then locked, and the trailing stance leg ceases contact with the ground. The model then transitions into the next single stance phase as shown in Figure 3. Prior to this transition, stance and swing legs are renamed, using the same single stance dynamics as the previous stride. All of our results in subsequent sections are based on numerical integration of these dynamics through one or more strides.

\section{Stability of walking with the AACG model}

\section{Periodic walking gaits}

We begin our analysis of AACG walking behaviours by identifying periodic walking gaits, corresponding to limit cycles, when all system parameters, including the precompressed 


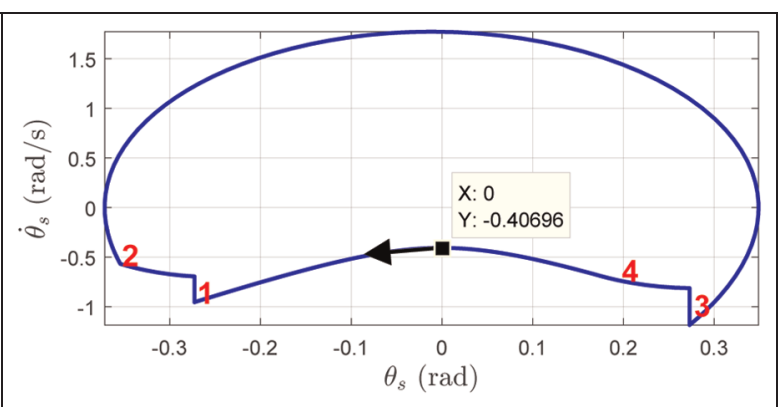

Figure 4. Phase space trajectories for an example periodic gait generated by the AACG model with $M=1 \mathrm{~kg}, m=0.01 \mathrm{~kg}, I=1 \mathrm{~m}$, $k=100 \mathrm{~N} / \mathrm{m}, r_{0}=0.05 \mathrm{~m}$. Only the states for one of the legs (leg A) are plotted, going through the stance phase at the bottom half and the swing phase at the top half. One cycle in the figure corresponds to two steps of the model. Events marked with I, 2, 3 and 4 correspond to the beginning of toe push-off for leg A, leg A liftoff, ground collision for leg B and the end of the toe push-off for leg $B$, respectively.

spring length, are fixed. For simplicity, let us define the initial swing and support as Leg A and Leg B, respectively. A projection of phase space trajectories corresponding to such a limit cycle are illustrated in Figure 4, where trajectories for only one leg (leg A) are shown. At the point marked with 1, the swing leg (leg B) collides with the ground, resulting in a discontinuous change in velocities. Subsequently, the ankle spring in leg A decompresses until point 2, which is when the ankle spring in leg A lifts off after injecting all of its stored energy into the model and reaches its rest length. Leg $A$ then becomes the new swing leg. The points marked 3 and 4 correspond to the collision of leg A with the ground, and the liftoff event for leg B completing the limit cycle.

A commonly used method for the identification and characterization of limit cycles in locomotory systems is Poincaré analysis, which relies on defining a co-dimension one subset of the state space, called the Poincare section, which transversally intersects all system trajectories. Successive intersections of system trajectories with this subset generate a discrete sequence, formally defined through the Poincare map (also called return map) that takes one intersection to the next. Fixed points of this map (and their stability) correspond to the presence (and stability) of limit cycles in the original system.

For the AACG model, we choose the Poincare section as the vertical, forward moving configurations of the supporting leg with $\theta_{s}=0$ and $\dot{\theta}_{n}>0$. This configuration corresponds to the highest point of the torso trajectory during the single support phase, which we call the apex point. As an example, the limit cycle illustrated in Figure 4 repeatedly intersects this section at $\theta_{s}=0 \mathrm{rad}, \dot{\theta}_{s}=-0.4069 \mathrm{rad} / \mathrm{s}, \theta_{n}=-0.0596 \mathrm{rad}$, $\dot{\theta}_{n}=1.7568 \mathrm{rad} / \mathrm{s}$, corresponding to a fixed point of the Poincaré map.

In addition to help identify limit cycles, the return map also allows the characterization of their stability properties through its linearization around fixed points. Eigenvalues of the resulting Jacobian can be used to characterize local stability properties for the limit cycles, with local asymptotic stability corresponding to all eigenvalues of the Jacobian falling within the unit circle.
In order to ensure the validity of the Poincare map, we check a number of fault conditions during our simulations. In particular, we discard and disregard trajectories that:

- locomote backwards with $\dot{\theta}_{s} \geq 0$;

- do not admit full extension of the ankle spring, with $d \leq r_{0}$;

- require the heel to go underground with $\dot{r}^{+}<0$;

- result in fault conditions such as the torso mass $M$ going underground, angle between the legs becoming unreasonably large or spring thrust being insufficient to ensure liftoff.

Elimination of such problematic cases ensures that all remaining trajectories of the AACG model pass transversally through the Poincare section at the apex point. More formally, let $x:=\left[\dot{\theta}_{s}, \theta_{n}, \dot{\theta}_{n}\right]^{\mathrm{T}}$ denote the state vector within the Poincaré section. Given Poincaré states $x_{i}$ and $x_{i+1}$ for the $i$ th and $(i+1)$ th apex points, respectively, the AACG return $\operatorname{map}, G: \mathbb{R} \times \mathrm{S}^{1} \times \mathbb{R} \rightarrow \mathbb{R} \times \mathrm{S}^{1} \times \mathbb{R}$, is defined as

$$
\mathbf{x}_{i+1}=\mathbf{G}\left(\mathbf{x}_{i}\right)
$$

We use a numerical computation of this apex return map to identify limit cycles for the AACG model together with their stability. In particular, limit cycles of the model correspond to fixed points $x^{*}$ of $\mathbf{G}$, defined through

$$
\mathbf{x}^{*}=\mathbf{G}\left(\mathbf{x}^{*}\right)
$$

Once we identify limit cycles in this fashion, we can determine their local stability by linearizing $G$ around the corresponding fixed point. This yields a local, linear approximation to the return map with

$$
\mathbf{x}_{i+1}-\left.\mathbf{x}^{*} \approx D \mathbf{G}\right|_{\mathbf{x}^{*}}\left(\mathbf{x}_{i}-\mathbf{x}^{*}\right)
$$

where $D \mathbf{G}$ denotes the Jacobian of $\mathbf{G}$. The limit cycle is then locally asymptotically stable if all eigenvalues of Jacobian matrix $\left.D \mathbf{G}\right|_{\mathbf{x}^{*}}$ are within the unit circle. Due to the hybrid nature of the AACG model and the complexity of its dynamics, there are no currently known closed-form expressions for the apex return map. Consequently, we use a second-order numerical approximation to compute the Jacobian matrix $\left.D \mathbf{G}\right|_{\mathbf{x}^{*}}$ for all of our simulations.

To illustrate, the limit cycle shown in Figure 4 corresponds to the fixed point $\mathbf{x}^{*}=[-0.4069,-0.0596,1.7568]$ for the apex return map, whose Jacobian matrix has eigenvalues $\lambda_{1}=-0.5715, \lambda_{2}=-0.1102, \lambda_{3}=0$. Since all eigenvalues are within the unit circle, this limit cycle was found to be locally asymptotically stable, which was also confirmed by the convergence of simulations starting from initial conditions close to the limit cycle.

Before we proceed with a more thorough characterization of limit cycles for the AACG model, we note that throughout all of our simulations, we observed one of the eigenvalues for all fixed points of the return map to be zero, meaning that the system recovers from perturbations along the associated eigenvector in a single step. For the example in Figure 4, this eigenvector is $\mathbf{x}_{0}=[0.0031,0.4389,0.8985]$. Figure 5 


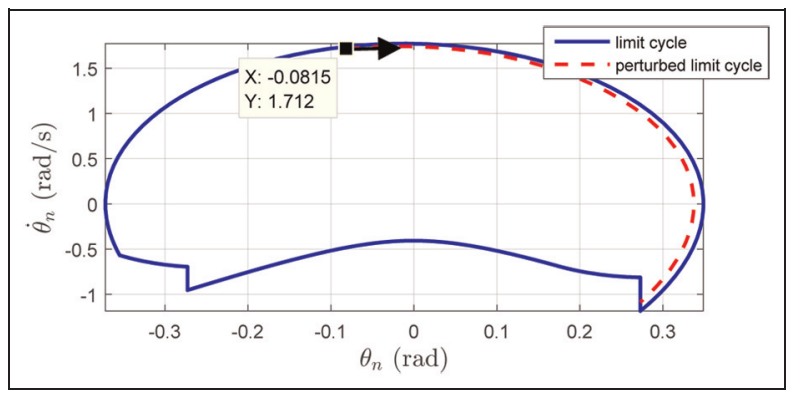

Figure 5. AACG trajectories resulting from a perturbation of the limit cycle in the direction of the eigenvector associated with the eigenvalue $\lambda=0$ for the apex return map. Model completely recovers from this perturbation immediately after toe collision.

illustrates AACG trajectories (dashed line) recover from a perturbation in this direction in a single step, right after the swing leg collision. An intuitive explanation for this phenomenon is offered by the fact that the initial swing leg position and velocity are coupled, for which there is a continuum of value pairs that result in the same collision configuration. Consequently, small perturbations which change these two DOF in a coupled fashion are rejected in a single step.

\section{Dependence of gait stability on system parameters}

In order to present a more complete picture of system behaviour, we now investigate the dependence of limit cycles and their stability on the amount of precompression $r_{0}$ in the ankle spring prior to its release. Physically, this precompression is often achieved with an actuator connected in series with the ankle spring, changing its rest length before its energy is released through the ankle joint. The left plot in Figure 6 presents the dependence of all three coordinates for the fixed points of the single-step apex return map on this precompression parameter. For all choices of precompression with $r_{0} \in[0,0.3](m)$, we have found two period-1 fixed points, both of which are unstable for mid-range parameter values, with one becoming stable for small and large precompression values. We have also shown cross sections of the basins of attraction associated with stable fixed point associated with $r_{0} \in[0,0.07](\mathrm{m})$ in the right plot of Figure 6 . These regions were obtained by fixing two coordinates on the stable limit cycle and varying the remaining coordinate in a broad range of initial conditions.

This is also shown in Figure 7, where the top plot illustrates all three eigenvalues associated with the fixed point that is initially stable and becomes unstable for mid-range choices of $r_{0}$. As we noted before, one of the eigenvalues is always zero, corresponding to the dependence of swing leg position and velocity prior to the collision. As the spring precompression increases, the remaining two eigenvalues first become a complex pair, then separate with one converging to zero, and the other crossing the stability threshold. To summarize, our results show that the uncontrolled AACG model exhibits period-1 stable limit cycles (self-stability) for a range of spring precompression values. This suggests that the use of SEA for the ankle joint of a walking platform is feasible with promising passive stability properties.

In the next section, we will explore period doubling behaviour outside regions where there are period-1 stable gaits. Subsequently, we will propose a feedback controller on the ankle precompression that forces the system to exhibit period1 stability for any desired forward velocity.

\section{Bifurcation regimes for periodic walking gaits}

In addition to the period-1 fixed points illustrated in Figure 6, it is also possible to investigate whether the system has limit cycles whose periodicity occurs in multiple steps. Dynamical systems often exhibit period doubling behaviour coincident with parametric or structural changes in the system behaviour, and such phenomena were previously shown to occur with passive dynamic walking models (Goswami et al., 1998).
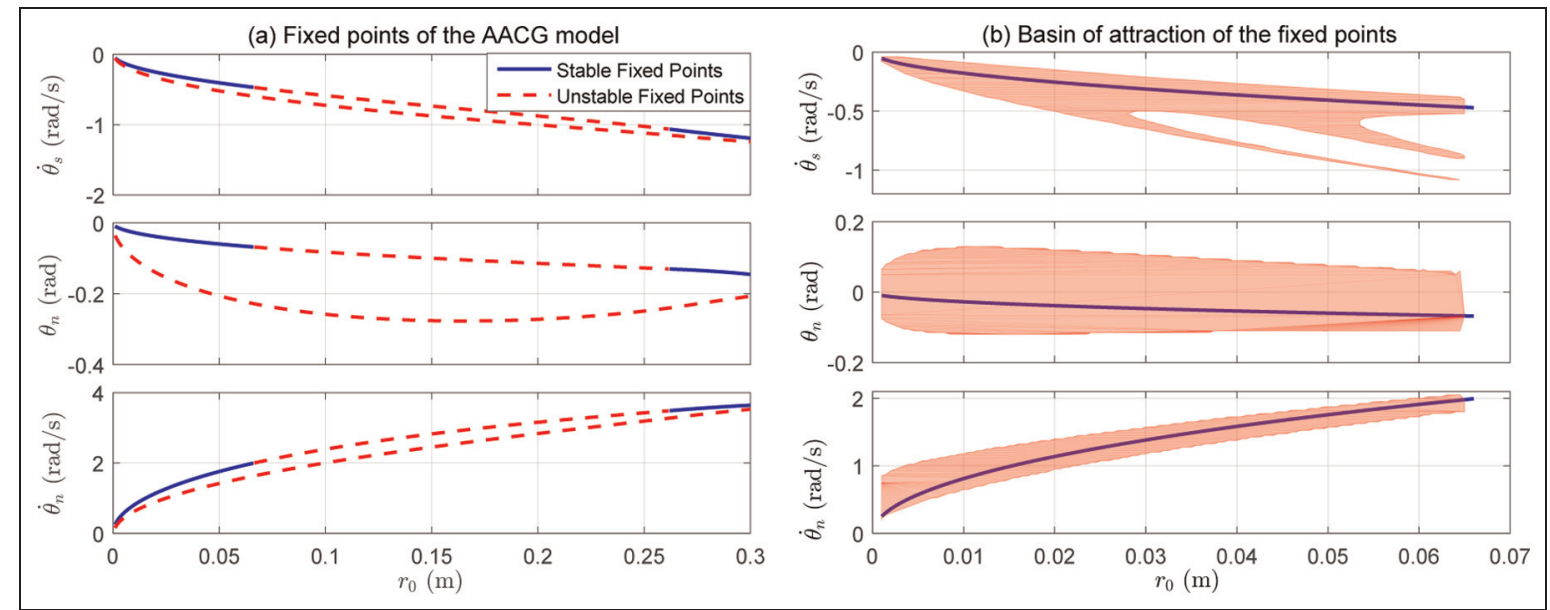

Figure 6. Both figures show fixed points of the AACG model with $k=100 \mathrm{~N} / \mathrm{m}$ as a function of ankle spring precompression. Solid and dashed plots show stable and unstable fixed points, respectively. Red regions in the right figure show cross sections of the basin of attraction for stable fixed points associated with $r_{0} \in[0,0.07] \mathrm{m}$. 


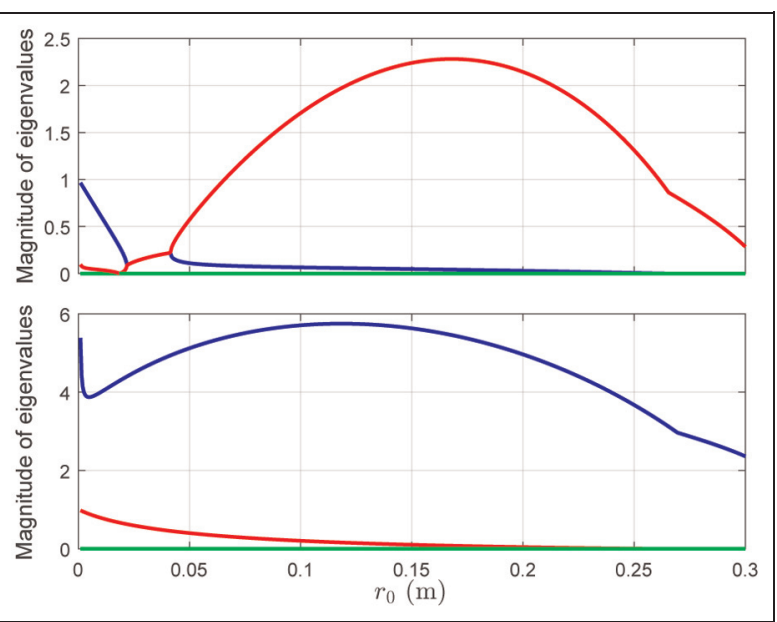

Figure 7. Eigenvalues of the linearized apex return map for the fixed point which is stable in a certain parameter range of the spring precompression (top) and the consistently unstable (bottom) fixed point as a function of the ankle spring precompression $r_{0}$ with $k=100 \mathrm{~N} / \mathrm{m}$.
In this section, we identify multi-step fixed points of the apex return map for the AACG model and show that it also exhibits period doubling behaviour, leading to sustained, period$2^{n}$, even chaotic walking patterns.

Stable period-1 AACG walking gaits observed for small values of $r_{0}$ eventually become unstable with increasing spring precompression. The parameter value at which one of the eigenvalues crosses outside the unit circle $\left(r_{0}=0.06573(\mathrm{~m})\right.$ for the example in Figure 6) marks the first bifurcation point, where period doubling occurs and a stable period-2 walking gait is introduced. This gait is increasingly asymmetric, consisting of a short and a long step. For larger values of spring precompression, short step becomes shorter and long step becomes longer. Further increases in the spring precompression parameter eventually destabilize this period-2 gait, leading to a stable period-4 gait. Figure 8 illustrates this period doubling behaviour, and Figure 10 shows phase space projections of limit cycles associated with period-2, period-4, period8 gaits and chaotic behaviour. Finally, Figure 9 illustrates the magnitudes of eigenvalues associated with period-1 to period4 gaits, showing that period doubling occurs when a particular

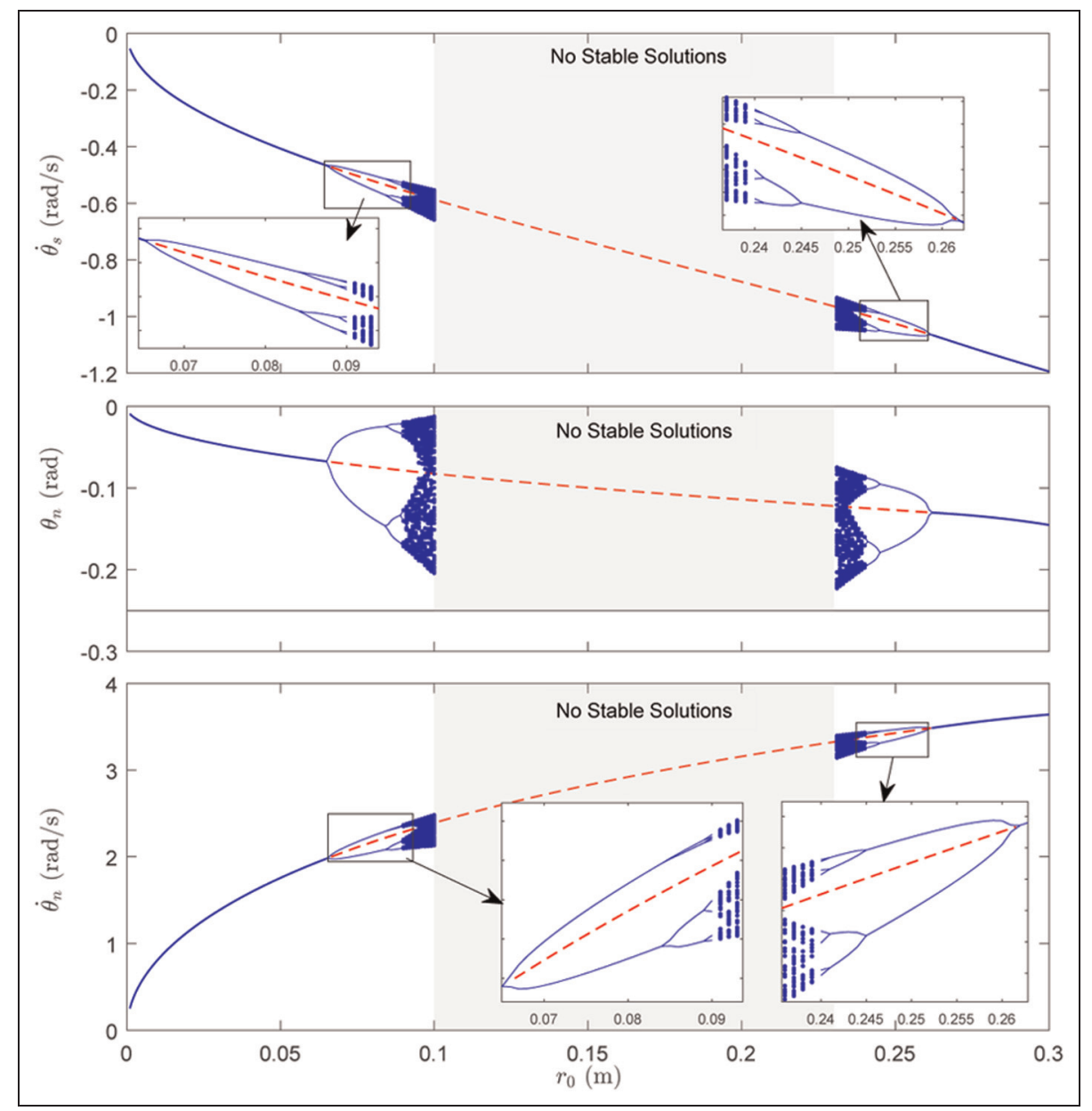

Figure 8. The dependence of fixed points of the AACG model on the spring precompression $r_{0}$. Solid blue plots show stable fixed points for different periodicities, whereas red dashed plots show unstable, period-I fixed points only. Regions where period doubling occurs up to period-8 gaits are magnified for clarity. 


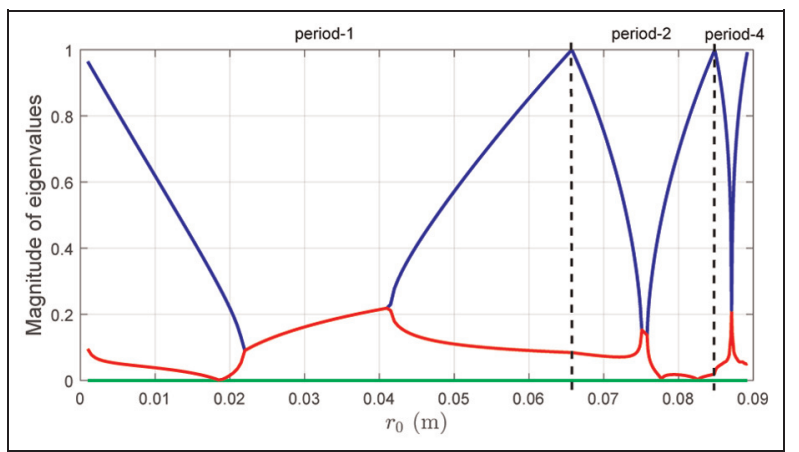

Figure 9. Magnitude of eigenvalues associated with stable gait periodicities up to 4 . Since the return map covers multiple steps for period-2 and period-4 regions, associated eigenvalues were plotted as $\lambda^{1 / 2}$ and $\lambda^{1 / 4}$, respectively, for continuity and better comparison with period-I eigenvalues. Eigenvalues beyond period- 4 were excluded since they are observed in a very narrow region.

gait becomes unstable with its largest magnitude eigenvalue crossing the unit circle.

We have also found, by evaluating parameter values at which period doubling occurs, listed in Table 1 for up to period-32 gaits, that the ratio of distances between successive bifurcations seem to converge to the Feigenbaum constant 4.669 (Strogatz, 2014).

Beyond $r_{0}>0.0905 \mathrm{~m}$, we were unable to find any periodic solutions, with each successive apex state different than previous ones even though the model kept walking indefinitely. This suggests a chaotic gait, where the locomotion is sustained, but no periodicity can be identified. For choices of spring precompression beyond this value, we evaluated the model for over 1000 steps, then recorded the last 100 apex states. Individual dots in Figure 8 plot each step from these runs, showing chaotic behaviour. Finally, beyond $r_{0}>0.1 \mathrm{~m}$ the AACG model could no longer sustain locomotion, with its return map having only unstable fixed points.

Interestingly, if $r_{0}$ is increased further, the AACG model eventually restarts being able to sustain locomotion. In particular, when the rest length reaches $r_{0}=0.2255 \mathrm{~m}$, a sustained but chaotic gait is observed, with no periodicity that we could identify. Further increases recover period- $2^{n}$ gaits, with parameter values at which the periodicity changes shown in Table 2. Period-1 gaits are observed again beyond $r_{0}=0.261079 \mathrm{~m}$. Similar to before, the ratio of the distances between successive bifurcations in this region also seem to converge to the Feigenbaum constant.

The occurrence of chaotic gaits beyond a certain spring precompression and the later recovery of symmetric period-1 stable gaits can be partially explained in terms of the energy input and losses in the AACG model. As the rest length increases, we inject more energy to the system and in response, the model attempts to walk faster causing previously stable fixed points to destabilize. This leads to a regime of period doubling bifurcations, chaos and eventually complete instability, which is consistent with existing literature on the PCG model (Garcia et al., 2000; Goswami et al., 1998).

Unlike those studies, however, the AACG model does not rely on the conservation of angular momentum to derive the collision map. Even though we can not use this principle due to the active double stance phase with both legs remaining on the ground, the recovery of periodic gait for larger values of the spring precompression can be explained with one of our previously described assumptions. In particular, beyond a certain value of precompression (and hence forward speed), the angle between the legs approaches $\pi / 2$, at which point the post-collision angular momentum vanishes since the body

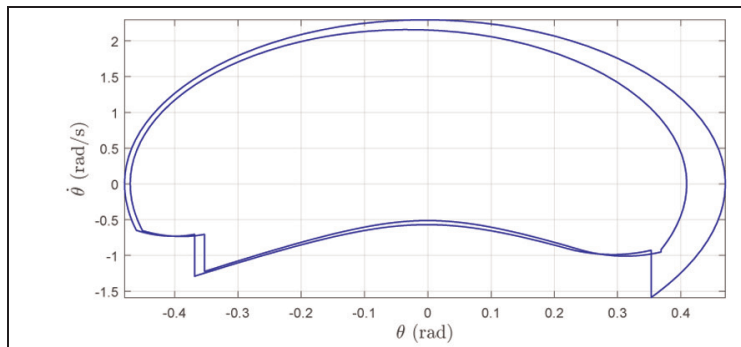

(a) Period-2 limit cycle at $r_{0}=0.084$.

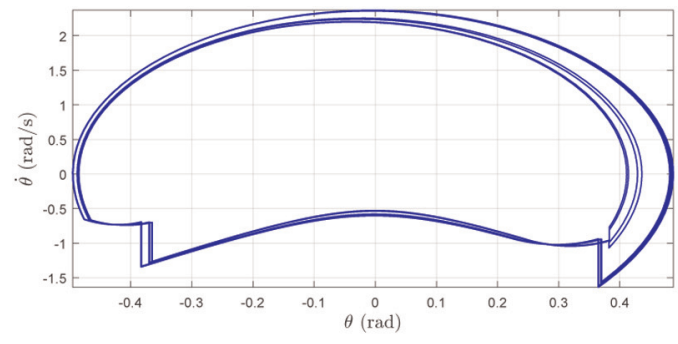

(c) Period-8 limit cycle at $r_{0}=0.09$.

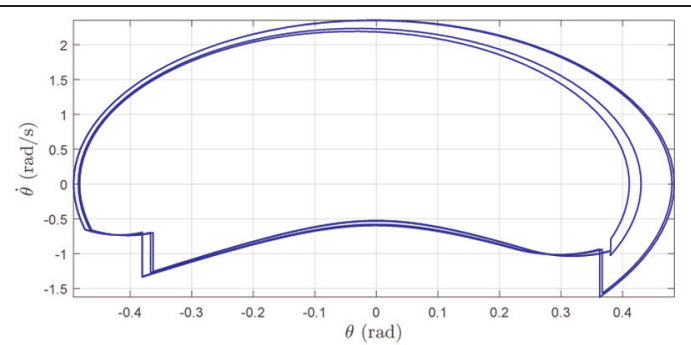

(b) Period-4 limit cycle at $r_{0}=0.089$.

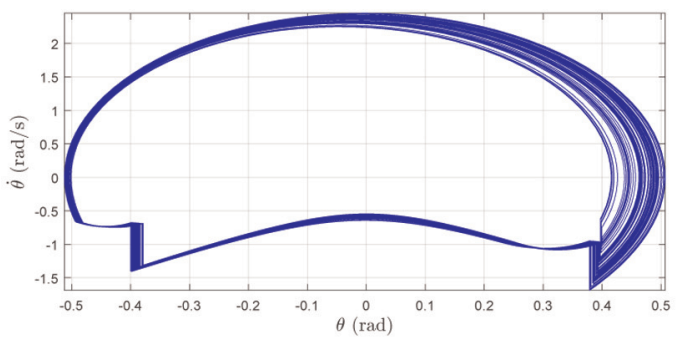

(d) Non-periodic, sustained walking at $r_{0}=0.098$.

Figure 10. Phase space projections of limit cycles with different periodicities and chaos for walking with the AACG model. 
Table I. Spring precompression parameter values at which period doubling occurs, together with ratios between successive parameter ranges for each period.

\begin{tabular}{llll}
\hline$n$ & New period & $r_{0}[n]$ & $\frac{r_{0}[n-1]-r_{0}[n-2]}{r_{0}[n]-r_{0}[n-1]}$ \\
\hline 1 & 2 & & - \\
2 & 4 & 0.06573 & - \\
3 & 8 & 0.08486 & 4.3379 \\
4 & 16 & 0.08927 & 4.5937 \\
5 & 32 & 0.09023 & 4.6154 \\
\hline
\end{tabular}

Table 2. Spring precompression parameter values at which changes in periodicity occur for large values of $r_{0}$, together with ratios between successive parameter ranges for each period doubling (coming backwards from $r_{0}=0.3 \mathrm{~m}$ ).

\begin{tabular}{llll}
\hline$n$ & New period & $r_{0}[n]$ & $\frac{r_{0}[n-1]-r_{0}[n-2]}{r_{0}[n]-r_{0}[n-1]}$ \\
\hline 1 & 2 & 0.26108 & - \\
2 & 4 & 0.24464 & - \\
3 & 8 & 0.240624 & 4.0895 \\
4 & 16 & 0.239728 & 4.47 \\
5 & 32 & 0.239534 & 4.6186 \\
\hline
\end{tabular}

velocity at the collision is then aligned with the colliding leg. This brings the model to a full stop after the collision. Moreover, since we assumed that the ankle acts in a unidirectional manner with $\dot{r}^{+} \geq 0$, inter-leg angles larger than $\pi / 2$ also have zero post-collision velocities. We believe that this property of the AACG model is what leads to the recovery of periodic gaits beyond a certain spring precompression, with the increase in collision losses compensating for the larger spring energy injected into the system. Figure 11 depicts the inter-leg angle, $\theta_{s}+\theta_{n}$, for the fixed points of the system over the entire range of the spring precompression, showing the disappearance and subsequent recovery of periodic gaits.

\section{Feedback control through the ankle spring}

As Figure 6 shows, a fixed choice of the spring precompression $r_{0}$ does not always yield a stable limit cycle for walking with the AACG model. It is, however, encouraging to note that period-1 fixed points exist for the entire range of this parameter, covering a wide range of forward velocities, which is directly related to the stance leg velocity $\dot{\theta}_{s}$ at apex (i.e. the top plot in Figure 6). If it were possible to achieve stable walking for this entire range, we would effectively obtain a walking model whose walking speed can be effectively controlled.

To this end, we propose to use feedback control on the ankle spring precompression, which can, in practice, be implemented using SEA, as evidenced by many successful legged platforms with similar designs (Au et al., 2009; Hitt et al., 2009). This can be accomplished through a simple proportional feedback law that preserves period-1 fixed points of Figure 6, while ensuring that one of the fixed points has all of

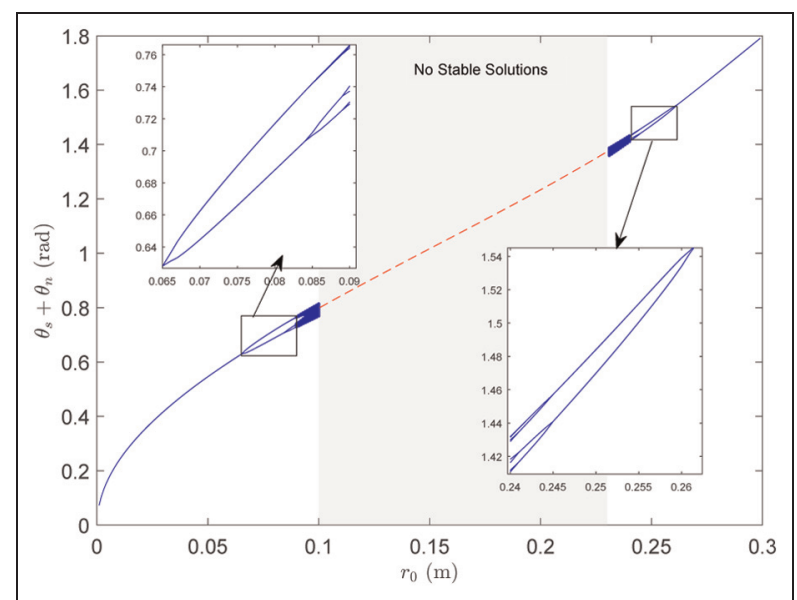

Figure II. Dependence of the inter-leg angle $\theta_{s}+\theta_{n}$ for the fixed points of the AACG model on the spring precompression parameter. Solid blue plots shows stable fixed points with different periodicities whereas the red dashed plot shows unstable period-I fixed points. Bifurcation regions up to period-8 gaits are magnified for a clearer view.

its eigenvalues within the unit circle. We begin by representing the stance leg angular velocity component of the fixed point which is stable in a certain parameter range of the spring precompression of Figure 6 in functional form as

$$
\dot{\theta}_{s}^{f p}=f\left(r_{0}\right)
$$

This function characterizes the fixed points and for our choice of dynamic parameters, it is monotonic in $r_{0}$ and is hence invertible. Consequently, for a desired velocity $\dot{\theta}_{s}^{*}$, we can find an open-loop spring precompression $r_{0}^{*}=f^{-1}\left(\dot{\theta}_{s}^{*}\right)$ that would yield a limit cycle coincident with the desired gait. This limit cycle, however, can be unstable. To address this problem, we propose the discrete-time feedback law

$$
r_{0}[k]=f^{-1}\left(\dot{\theta}_{s}^{*}\right)-k_{p}\left(\dot{\theta}_{s}^{*}[k]-\dot{\theta}_{s}[k]\right)
$$

where $k=0,1,2, \ldots, n$ are the discrete time instances at apex points. This law adjusts the spring precompression based on the measured error in the stance leg velocity at apex through negative feedback. As shown in Figure 12, increasing the gain $k_{p}$ allows us to adjust the eigenvalues associated with the period-1 limit cycle to lie within the unit circle, effectively stabilizing otherwise unstable fixed points for the AACG model. Since the feedback law (16) leaves fixed points of the return map unchanged, it allows exact, stable control of walking at a desired apex velocity. Fixed points with larger periodicities seem to disappear under this regime for large enough values of $k_{p}$.

\section{Conclusions}

In this study, we focused on a planar, dynamic walking model with active ankle actuation through SEA. Our model summarizes the action of the normally rotary ankle joint with a simplified prismatic joint. The spring situated in the ankle joint of the stance leg is activated immediately following the collision of the swing leg with the ground, modelling toe 

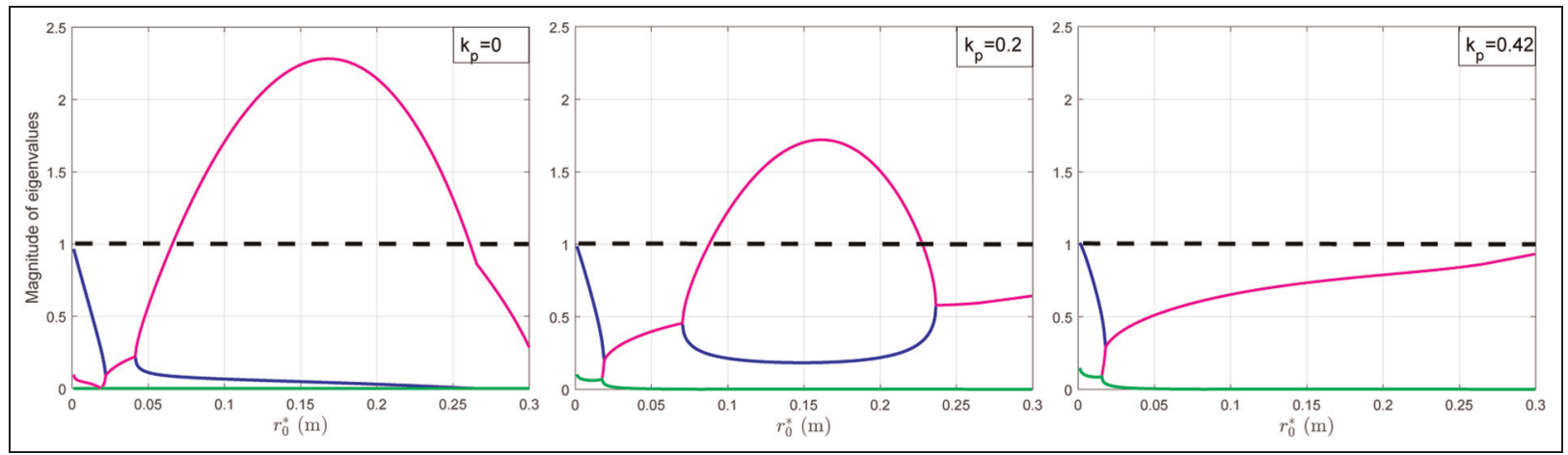

Figure 12. The dependence of AACG fixed point stability on the feedback gain $k_{p}$. The left plot shows the eigenvalues of the uncontrolled system, with the middle and right plots showing the eigenvalues with $k_{p}=0.02$ and $k_{p}=0.042$, respectively. The dashed lines mark the unit magnitude threshold for the eigenvalues.

push-off similar to human walking. We derived equations of motion associated with all locomotory phases for this model, namely single stance dynamics, impulsive collision map and double stance dynamics, and we numerically implemented the resulting hybrid dynamics. Subsequently, we defined a Poincaré section at the highest point of the stance phase and used numerical methods to compute the associated Poincaré return map and its Jacobian. Fixed points of this map were then used to identify limit cycles of this system, with the eigenvalues of the corresponding Jacobian matrix leading to a careful characterization of limit cycle stability. Our results showed that the AACG model we proposed in this study exhibits locally asymptotically stable limit cycles corresponding to feasible, sustained walking gaits on flat ground.

We extended our analysis by investigating the dependence of gait stability on the spring precompression realized prior to the swing leg collision. The increase in this precompression parameter eventually destabilizes period-1 limit cycles and introduces a regime of period doubling bifurcations. Period doublings evolve into non-periodic but sustained walking gaits and further increase of the precompression parameter results in unsustainable locomotion. We observed an interesting recovery of walking gaits for larger values of the spring precompression where a sustained but chaotic gait is reintroduced. Possible explanation for this behaviour is due to the use of a unidirectional ankle spring to model the effects of the heel in our collision map. Further increase in the precompression parameter yields period- $2^{n}$ through period-1 gaits. For both regions of bifurcating behaviour, we showed that the ratio of distances between successive period doubling values converge to the Feigenbaum constant.

Our results revealed that walking with a fixed spring precompression at each step does not always generate stable, period-1 walking behaviour. Consequently, we implemented feedback control adjusting the ankle spring precompression, which enabled us to stabilize the otherwise unstable, period-1 fixed points, allowing explicit, stable control on the walking speed for the model.

One of our long-term motivations in this research is our goal of implementing stable and energy efficient actuation methods for AAFO systems that can also be integrated with lower-extremity exoskeletons. Such platforms allow people with mobility disorders to stand and walk. In this context, existing research focuses on restoring only the functionalities of the knee and hip joints and ignore ankle joint despite its key role for the energetics and stability of walking. This lack of ankle actuation limits energetic efficiency for these platforms, and results in unnatural walking patterns. Our results provide a step in understanding parametric design and stability tradeoffs in achieving dynamic walking with SEA on the ankle. We eventually hope to use our methods in both the design and control of experimental instantiations of AAFO systems.

In the near future, we will explore generalizations of our results to nonzero inclinations and rough terrain. Subsequently, we will explore possible efficiency gains by adjusting the timing of toe push-off, that might enable the model to achieve approximate ground speed matching for the swing toe prior to its collision with the ground. In the long term, we hope to first construct simple, experimental instantiations of the model to verify our stability results and controllers, eventually leading to integration with robotic orthosis platforms.

\section{Funding}

The author(s) disclosed receipt of the following financial support for the research, authorship, and/or publication of this article: This research was funded by The Scientific and Technological Research Council of Turkey (TUBITAK) project number 114E277 and the Ministry of Science, Industry and Technology of Turkey project number 0067.STZ.2013-1.

\section{References}

Ahn J and Hogan N (2012) A simple state-determined model reproduces entrainment and phase-locking of human walking. PloS One 7(11): e47963.

Asano F, Luo ZW and Hyon S (2005) Parametric excitation mechanisms for dynamic bipedal walking. In: Proceedings of the IEEE international conference on robotics and automation, pp.609-615.

Au SK, Weber J and Herr H (2009) Powered ankle-foot prosthesis improves walking metabolic economy. IEEE Transactions on Robotics 25(1): 51-66.

Bhounsule PA (2015) Control of a compass gait walker based on energy regulation using ankle push-off and foot placement. Robotica 33(6): 1314-1324.

Blaya J and Herr H (2004) Adaptive control of a variable-impedance ankle-foot orthosis to assist drop-foot gait. IEEE Transactions on Neural Systems and Rehabilitation 12(1): 24-31. 
Byl K and Tedrake R (2008) Approximate optimal control of the compass gait on rough terrain. In: Proceedings of the IEEE international conference on robotics and automation. pp.1258-1263.

Collins SH, Wisse M and Ruina A (2001) A three-dimensional passive-dynamic walking robot with two legs and knees. The International Journal of Robotics Research 20(7): 607-615.

Collins SH and Ruina A (2005) A bipedal walking robot with efficient and human-like gait. In: Proceedings of the IEEE international conference on robotics and automation. pp.1983-1988.

Garcia M, Ruina A, Coleman M, et al. (1996) Passive-dynamic models of human gait. In: Proceedings of the conference on biomechanics and neural control of movement, pp.32-33.

Garcia M, Chatterjee A, Ruina A, et al. (1998) The simplest walking model: Stability, complexity, and scaling. ASME Journal of Biomechanical Engineering 120: 281-288.

Garcia M, Chatterjee A and Ruina A (2000) Efficiency, speed, and scaling of two-dimensional passive-dynamic walking. Dynamical Systems 15(2): 75-99.

Ghorbani R (2008) On controllable stiffness bipedal walking. PhD Thesis, The University of Manitoba, Canada.

Goswami A, Espiau B and Keramane A (1996) Limit cycles and their stability in a passive bipedal gait. In: Proceedings of the IEEE international conference on robotics and automation, 1, pp.246-251.

Goswami A, Espiau B and Keramane A (1997) Limit cycles in a passive compass gait biped and passivity-mimicking control laws. Autonomous Robots 4(3): 273-286.

Goswami A, Thuilot B and Espiau B (1998) A study of the passive gait of a compass-like biped robot: Symmetry and chaos. The International Journal of Robotics Research 17(12): 1282-1301.

Grizzle JW, Abba G and Plestan F (2001) Asymptotically stable walking for biped robots: Analysis via systems with impulse effects. IEEE Transactions on Automatic Control 46(1): 51-64.

Hitt J, Oymagil AM, Sugar T, et al. (2007) Dynamically controlled ankle-foot orthosis (DCO)with regenerative kinetics: Incrementally attaining user portability. In: Proceedings of the IEEE international conference on robotics and automation, pp.1541-1546.

Hitt J, Sugar T, Holgate M, et al. (2009) Robotic transtibial prosthesis with biomechanical energy regeneration. Industrial Robot: An International Journal 36(5): 441-447.

Hobbelen D and Wisse M (2008a) Ankle actuation for limit cycle walkers. The International Journal of Robotics Research 27(6): 709-735.

Hobbelen D, De Boer T and Wisse M (2008b) System overview of bipedal robots flame and tulip: Tailor-made for limit cycle walking. In: Proceedings of the IEEE/RSJ international conference on intelligent robots and systems, pp.2486-2491.

Iida F and Tedrake R (2009) Minimalistic control of a compass gait robot in rough terrain. In: Proceedings of the IEEE international conference on robotics and automation, pp.1985-1990.

Kerimoglu D, Morgul O and Saranli U (2015) Stability of a compass gait walking model with series elastic ankle actuation. In: Proceedings of the international conference on advanced robotics, pp.351-356.

Kuo AD (2002) Energetics of actively powered locomotion using the simplest walking model. Journal of Biomechanical Engineering 124(1): 113-120.

Maykranz D and Seyfarth A (2014) Compliant ankle function results in landing-take off asymmetry in legged locomotion. Journal of Theoretical Biology 349: 44-49.

McGeer T (1990) Passive dynamic walking. International Journal of Robotics Research 9(2): 62-82.

Spong MW and Bhatia G (2003) Further results on control of the compass gait biped. In: Proceedings of the IEEE/RSJ international conference on intelligent robots and systems, vol. 2, pp.1933-1938.
Strogatz SH (2014) Nonlinear Dynamics and Chaos: With Applications to Physics, Biology, Chemistry, and Engineering. Boulder, CO: Westview press.

Tang C, Yan G, Lin Z, et al. (2014) Stable walking of 3d compasslike biped robot with underactuated ankles using discrete transverse linearization. Transactions of the Institute of Measurement and Control 37(9): 1074-1083.

Tedrake R, Zhang TW, Fong MF, et al. (2004) Actuating a simple 3d passive dynamic walker. In: Proceedings of the IEEE international conference on robotics and automation, vol. 5, pp.4656-4661.

Van Der Linde RQ (1998) Active leg compliance for passive walking. In: Proceedings of the IEEE international conference on robotics and automation, vol. 3, pp.2339-2344.

Wang Z, Yan G, Tang C, et al. (2015) Coupling mechanical design and control design for energy-efficient and stable walking of a compass-like biped. Transactions of the Institute of Measurement and Control 38(3): 253-265.

Zelik KE, Huang TWP, Adamczyk PG, et al. (2014) The role of series ankle elasticity in bipedal walking. Journal of Theoretical Biology 346: $75-85$.

\section{Appendix}

\section{General collision map for the AACG model}

In this section, we present detailed expressions for the collision map, mapping velocities before and after the collision event with $\dot{\mathbf{q}}_{c}^{+}=\mathbf{H}_{c}\left(\mathbf{q}_{c}^{-}\right) \dot{\mathbf{q}}_{c}^{-}$. We define

$$
\boldsymbol{H}_{\boldsymbol{c}}:=\frac{1}{H_{d}}\left[\begin{array}{lll}
H_{11} & H_{12} & H_{13} \\
H_{21} & H_{22} & H_{23} \\
H_{31} & H_{32} & H_{33}
\end{array}\right]
$$

whose components are obtained from (10) to yield

$$
\begin{aligned}
H_{d}:=- & l^{2}(7 m+8 M)-16 l(m+M) r^{-}-2(5 m+4 M)\left(r^{-}\right)^{2} \\
& +m l\left(3 l+4 r^{-}\right) \cos \left(2\left(\theta_{s}^{-}-\theta_{n}^{-}\right)\right)
\end{aligned}
$$

and

$$
\begin{aligned}
H_{11}: & =-2 \cos \left(\theta_{s}^{-}-\theta_{n}^{-}\right)^{2}\left(l^{2}(3 m+4 M)+8 l(m+M) r^{-}\right. \\
& \left.+2(3 m+2 M)\left(r^{-}\right)^{2}\right) \\
H_{12}: & =2 m l \cos \left(\theta_{s}^{-}-\theta_{n}^{-}\right)\left(l+r^{-}\right) \\
H_{13}:= & -(6 m+4 M)\left(l+r^{-}\right) \sin \left(2\left(\theta_{s}^{-}-\theta_{n}^{-}\right)\right) \\
H_{21}:= & -2 \cos \left(\theta_{s}^{-}-\theta_{n}^{-}\right)\left(l+r^{-}\right)\left(l^{2}(3 m+4 M)\right. \\
& \left.+8 l(m+M) r^{-}+(6 m+4 M)\left(r^{-}\right)^{2}\right) / l \\
H_{22}: & =2 m\left(l+r^{-}\right)^{2} \\
H_{23}: & =-4(3 m+2 M)\left(l+r^{-}\right)^{2} \sin \left(\theta_{s}^{-}-\theta_{n}^{-}\right) / l \\
H_{31}: & =-\left(l+r^{-}\right) \sin \left(2\left(\theta_{s}^{-}-\theta_{n}^{-}\right)\right)\left(l^{2}(3 m+4 M)\right. \\
& \left.+(6 m+4 M)\left(r^{-}\right)^{2}\right) \\
H_{32}: & =2 m l\left(l+r^{-}\right)^{2} \sin \left(\theta_{s}^{-}-\theta_{n}^{-}\right) \\
H_{33}: & =-(12 m+8 M)\left(l+r^{-}\right)^{2} \sin \left(\theta_{s}^{-}-\theta_{n}^{-}\right)^{2}
\end{aligned}
$$

This general form of the collision map is further simplified for the AACG model, whose single stance ends with $r^{-}=0$ and $\dot{r}^{-}=0$ to yield the simplified map of (11). 